Received: 12 January 2017

Accepted: 13 April 2017

Published online: 24 May 2017

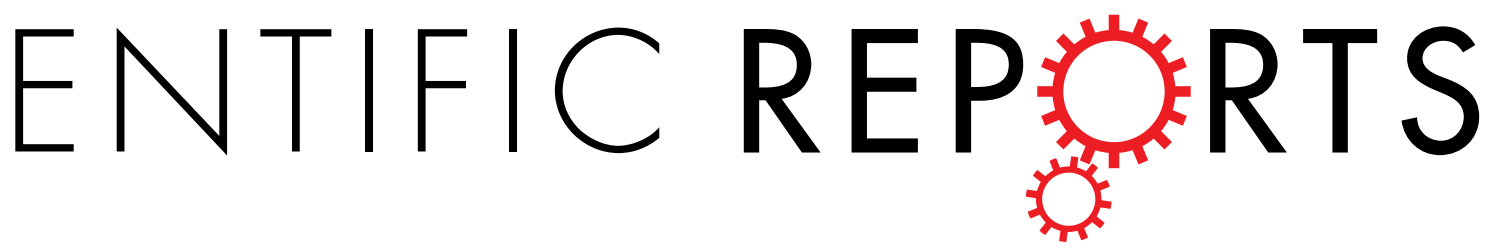

\title{
A core extended naphtalene diimide G-quadruplex ligand potently inhibits herpes simplex virus 1 replication
}

Sara Callegaro ${ }^{1}$, Rosalba Perrone ${ }^{1}$, Matteo Scalabrin ${ }^{1}$, Filippo Doria ${ }^{2}$, Giorgio Palù ${ }^{1}{ }^{1} \&$ Sara N. Richter ${ }^{1}$

G-quadruplexes (G4s) are nucleic acids secondary structures, epigenetic regulators in cells and viruses. In herpes simplex virus 1 (HSV-1)-infected cells, G4s are massively present during viral replication. We here aimed at investigating the possibility to target the HSV-1 G4s by a core extended naphtalene diimide (c-exNDI) G4 ligand. Biophysical and biomolecular analysis proved that c-exNDI stabilized the HSV-1 G4s in a concentration dependent manner. In MS competition assays, c-exNDI preferentially recognized HSV-1 G4s over cellular telomeric G4s, the most represented G4s within cells; other less abundant cellular G4s were also recognized. Treatment of HSV-1 infected cells with c-exNDI at low nanomolar concentrations induced significant virus inhibition with no cytotoxicity. The mechanism of action was ascribed to G4-mediated inhibition of viral DNA replication, with consequent impairment of viral genes transcription. Our data suggest that the observed potent antiviral activity and low cytotoxicity mainly depend on a combination of c-exNDI affinity for HSV-1 G4s and their massive presence during infection. HSV-1 G4s may thus represent new effective antiviral targets: the fact that no current antiherpetic drug exploits them and their presence at the viral genome, responsible for both active and latent HSV infections, makes them particularly attracting.

G-quadruplexes (G4s) are nucleic acids secondary structures that may form in single-stranded G-rich sequences under physiological conditions ${ }^{1}$. Four Gs bind via Hoogsteen-type hydrogen bonds base-pairing to yield $\mathrm{G}$-quartets, which stack to form the G4. The presence of $\mathrm{K}^{+}$cations specifically supports $\mathrm{G} 4$ formation and stability $^{2}$. Based on the strand orientation, G4s can adopt three main topologies: parallel, antiparallel, and hybrid-type structures. In eukaryotes, G4s have been shown to be involved in key regulatory roles, including transcriptional regulation of gene promoters and enhancers, translation, chromatin epigenetic regulation, DNA recombination $^{3-7}$. Expansion of G-quadruplex-forming motifs has been associated with relevant human neurological disorders $^{4,8,9}$. Formation of G4s in vivo has been consolidated by the discovery of cellular proteins that specifically recognize $\mathrm{G} 4 \mathrm{~s}^{10,11}$ and the development of $\mathrm{G} 4$ specific antibodies ${ }^{12,13}$.

Recently, the presence of G4s in viruses and their involvement in virus key steps has been provided ${ }^{14}$. G4s have been implicated in pathogenic mechanisms of the human immunodeficiency virus, where functionally significant G4s have been identified ${ }^{5,11,15-17}$ and stabilized by G4 ligands with consequent antiviral effects ${ }^{5,18,19}$. G4s have been reported in the SARS coronavirus ${ }^{20}$, the human papilloma, Zika, Ebola and hepatitis C virus genome ${ }^{21-24}$. Among herpesviruses, RNA G4s have been implicated in the regulation of DNA replication and translation of the Epstein-Barr virus ${ }^{25,26}$. We have shown that the herpes simplex virus 1 (HSV-1) possesses several repeats of important G4-forming sequences, which could be stabilized by a G4 ligand with inhibition of viral DNA replication $^{27}$. In addition, HSV-1 G4s, visualized with the aid of a G4-specific antibody in infected cells ${ }^{12}$, were shown to form massively in the cell nucleus, peak during viral replication and localize according to the viral genome intracellular movements ${ }^{28}$.

The involvement of G4 structures in several human diseases propelled the development of small molecules directed against $\mathrm{G} 4 \mathrm{~s}^{7,29}$. However, only very few have been tested against viruses, i.e. BRACO-19 (HIV-1, EBV and

${ }^{1}$ Department of Molecular Medicine, University of Padua, via Gabelli 63, 35121, Padua, Italy. ${ }^{2}$ Department of Chemistry, University of Pavia, V.le Taramelli 10, 27100, Pavia, Italy. Correspondence and requests for materials should be addressed to S.N.R. (email: sara.richter@unipd.it) 


\begin{tabular}{|c|c|c|c|c|c|c|}
\hline \multicolumn{7}{|c|}{ Melting temperature } \\
\hline \multirow{2}{*}{$\begin{array}{l}\text { G4 sequence } \\
\text { c-exNDI }\end{array}$} & \multicolumn{2}{|l|}{$\mathrm{KCl} 0\left({ }^{\circ} \mathrm{C}\right)$} & \multirow[t]{2}{*}{$\Delta \mathrm{T}_{\mathrm{m}}\left({ }^{\circ} \mathrm{C}\right)$} & \multicolumn{2}{|c|}{$\mathrm{KCl} 100 \mathrm{mM}\left({ }^{\circ} \mathrm{C}\right)$} & \multirow[t]{2}{*}{$\Delta \mathrm{T}_{\mathrm{m}}\left({ }^{\circ} \mathrm{C}\right)$} \\
\hline & - & + & & - & + & \\
\hline gp054a & nd & nd & - & $64.3 \pm 0.84$ & $79.5 \pm 0.61$ & 15.2 \\
\hline un2 & $50.6 \pm 0.53$ & $56.8 \pm 0.62$ & 6.2 & $>90$ & $>90$ & - \\
\hline un3 & nd & nd & - & $65.1 \pm 3.03$ & $>90$ & $>24.9$ \\
\hline
\end{tabular}

Table 1. Melting temperatures $\left(\mathrm{T}_{\mathrm{m}},{ }^{\circ} \mathrm{C}\right)$ of HSV-1 G4 folding sequences measured by CD in the absence or presence $(100 \mathrm{mM})$ of $\mathrm{KCl}$. Each sequence $(4 \mu \mathrm{M})$ was analyzed in the absence or presence of c-exNDI $(16 \mu \mathrm{M})$. nd stands for "not detected".

HSV-1), pyridostatin (EBV) and Core- Extended Naphtalen Diimide compounds (c-exNDIs) (HIV-1) 5, 18, 19, 25, 26. Since no definitive drugs have been found against most viral infections, there is an obvious need for new more active compounds.

C-exNDI derivatives have been shown to display fair selectivity towards the HIV-1 G4s that form in the LTR viral promoter versus cellular $\mathrm{G} 4 \mathrm{~s}^{19}$. This selectivity rests on the preferred recognition of the loop regions of the HIV-1 vs cellular G4s. We here sought to investigate if the best anti-HIV-1 compound of the series, c-exNDI 2 in our previous work ${ }^{19}$, displayed also anti-HSV-1 activity and to test its mechanism of action.

We found that c-exNDI was able to bind and stabilize the HSV-1 G4 forming sequences in a concentration dependent manner. Treatment of HSV-1 infected cells with c-exNDI induced a complete inhibition of the virus at low nanomolar concentration, with a mechanism of action directed toward viral DNA replication. Inhibition of viral DNA replication impaired viral genes transcription resulting in an effective antiherpetic effect. MS competition assays demonstrated that c-exNDI preferentially binds HSV-1 G4s over cellular telomeric G4, the most represented G4s within cells, suggesting that the observed preferential antiviral activity vs cytotoxicity is mediated by both the higher amount of HSV-1 G4s in the cell and their higher affinity for c-exNDI.

\section{Results}

The G4 ligand c-exNDI highly stabilizes the HSV-1 G4s. We have previously shown that c-exNDIs, compounds that were specifically developed against HIV-1 G4s, were indeed able to selectively recognize and bind with high affinity the HIV-1 G4 sequences ${ }^{19}$. The human telomeric G4-folded sequence and the G4s forming in the promoters of some oncogenes were also bound, albeit with lower affinity. To test the possibility to expand their antiviral activity, we here evaluated the ability of the lead member of the c-exNDIs family, c-exNDI 2 in ${ }^{19}$ (herein called c-exNDI) to interact also with the G4s forming in the terminal repeats of HSV-1.

Three sequences were considered: two sequences forming a four-stacked-G-quartet structure (un2 and gp054a) and one forming a three-G-quartet G4 (un3) ${ }^{27}$. Stability and conformation changes upon addition of c-exNDI were detected by circular dichroism (CD).

In $100 \mathrm{mM} \mathrm{K}^{+}$, c-exNDI stabilized $u n 3$ and $g p 054 a$ by $>24.9^{\circ} \mathrm{C}$ and $15.2^{\circ} \mathrm{C}$, respectively (Table 1 ), and induced a slight change in the oligonucleotide G4 conformation at $20^{\circ} \mathrm{C}$ (Fig. 1a,b). In the case of $g p 054 a$, during thermal unfolding, the presence of the compound showed an increased stabilization of an alternative parallel-like conformation (Fig. 1b). Un2 G4 displayed a starting $\mathrm{T}_{\mathrm{m}}$ above $90^{\circ} \mathrm{C}$ in 100,20 and $5 \mathrm{mM} \mathrm{K}^{+}$, therefore no appreciable $\Delta \mathrm{T}_{\mathrm{m}}$ value could be obtained. However, a mild CD spectrum variation of un 2 upon addition of c-exNDI indicated interaction (Fig. 1c): in addition, in the absence of $\mathrm{K}^{+}$, an appreciable $\Delta \mathrm{T}_{\mathrm{m}}$ was obtained with c-exNDI (Table 1). These data indicate that c-exNDI is able to effectively bind and stabilize all HSV-1 G4s.

To confirm the increased stability of the viral G4s in the presence of the compound, a Taq polymerase stop assay was set up. Templates corresponding to $u n 2, u n 3$ and $g p 054 a$ sequences (Table S1) were annealed to a primer and incubated with Taq polymerase for $30 \mathrm{~min}$ at $60^{\circ} \mathrm{C}$. A sequence unable to fold into $\mathrm{G} 4$ was alongside assayed as negative control. In the absence of $\mathrm{K}^{+}$, un 2 and gp054a displayed a marked stop site corresponding to the first G of the most $3^{\prime}$ G-tract (Fig. 2a, lanes 1 un 2 and gp054), indicating stable G4 folding. The stop site increased upon addition of $\mathrm{K}^{+}$and c-exNDI (Fig. 2a, lanes 2-5 un2 and gp054); moreover, in the presence of the compound the full-length amplified product sharply decreased, indicating effective stabilization of un 2 and $g p 054 a \mathrm{G} 4 \mathrm{~s}$ and thus inhibition of polymerase progression (Fig. 2a, lanes 3-5 un2 and gp054). In the absence of $\mathrm{K}^{+}$no stop site was observed in the $u n 3$ sequence, which thus did not form G4 in these conditions (Fig. 2a, lane 1 un3); upon addition of $\mathrm{K}^{+}$and c-exNDI, a marked stop became visible at the most $3^{\prime}$ G-tract (Fig. 2a, lanes 2-5 un3), indicating effective stabilization also of this template. Quantification of the stop sites indicated a similar degree of polymerase stalling induced by the compound in the three G4-forming sequences (Fig. 2b).

To check the selectivity of c-exNDI for HSV-1 G4s, mass spectrometry (MS) competition was performed. Selected competitor G4s were hTel, c-myc and c-kit2 G4s, which correspond to the G4-forming sequences of the human telomeric repeat, the promoter of the c-myc oncogene and c-kit protooncogene ${ }^{30,31}$, and HIV-1 LTR-III G4, the HIV-1 G4 previously shown to be selectively bound with high affinity by c-exNDI ${ }^{5,19}$. In each case, HIV-1 LTR-III G4s were preferred over HSV-1 G4s, data that confirm c-exNDI selectivity for HIV-1 G4s ${ }^{19}$ (Table 2). C-myc G4 was also always preferred and c-kit2 G4 was preferred vs HSV-1 un2 and gp054a. In contrast, HSV-1 un 2 and un $3 \mathrm{G} 4 \mathrm{~s}$ were preferred over hTel. Gp054a was generally less preferentially bound over all the other analysed sequences. The measured selectivity did not depend on the starting stability of the competing G4s: in fact, in $100 \mathrm{mM} \mathrm{K}^{+}$, preferred G4s such as LTR-III $\left(\mathrm{T}_{\mathrm{m}} 52.9 \pm 0.2^{\circ} \mathrm{C}\right)$ and c-myc $\left(\mathrm{T}_{\mathrm{m}}>90^{\circ} \mathrm{C}\right)$ had $\mathrm{T}_{\mathrm{m}}$ values both lower and higher, respectively, than HSV-1 G4s (Table 1); in contrast, non-preferred G4s had similar $\mathrm{T}_{\mathrm{m}}$ values (hTel21 $68.6 \pm 0.2$, c-kit2 $72.9 \pm 1.1^{\circ} \mathrm{C}$ ) (Table 1$)$. 

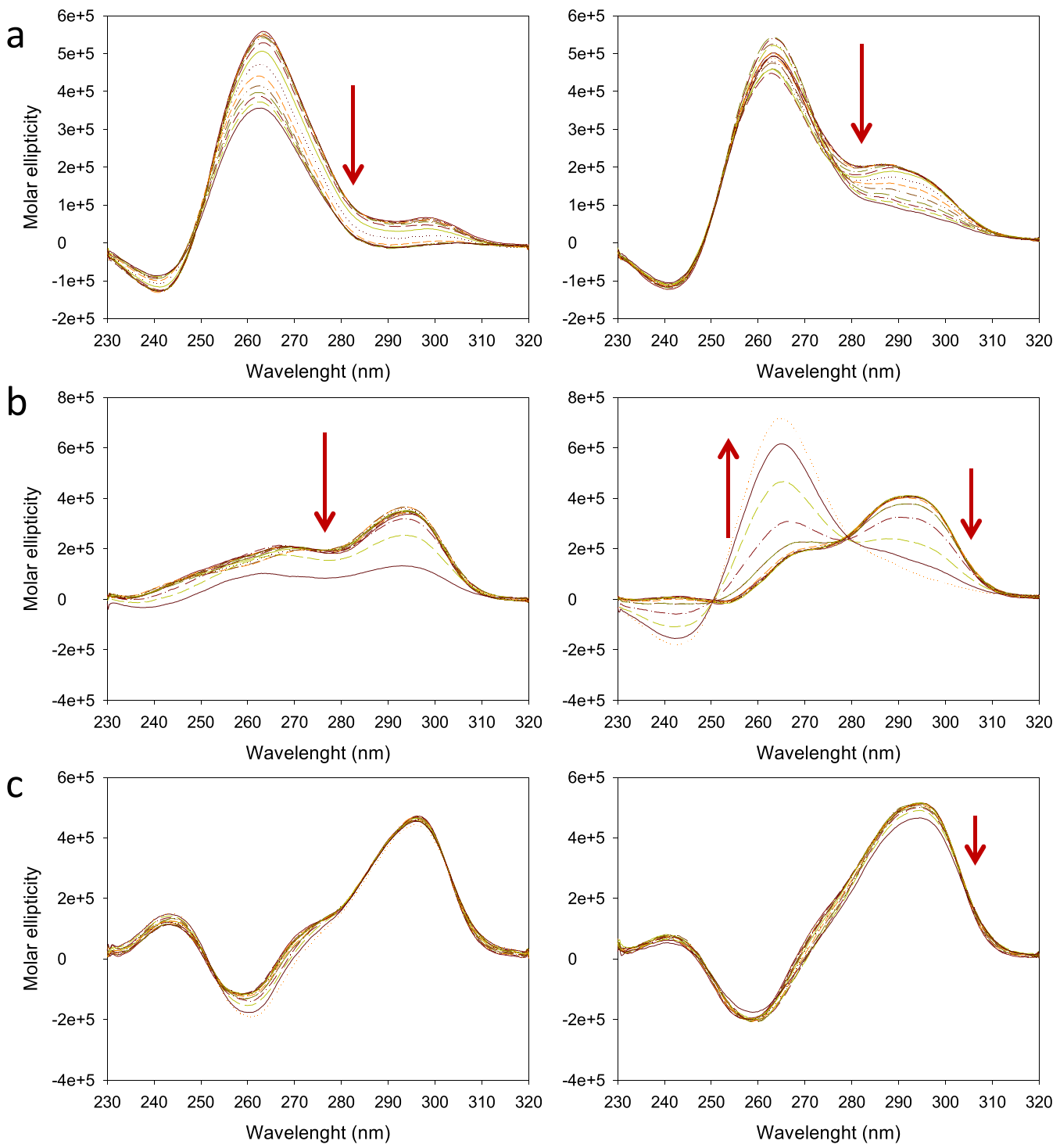

Figure 1. Thermal unfolding of un3 parallel G4 (a), gp054a hybrid G4 (b), un2 antiparallel G4 (c) $(4 \mu \mathrm{M})$ in $100 \mathrm{mM} \mathrm{K}^{+}$in the absence (left panel) or presence (right panel) of $16 \mu \mathrm{Mc}$-exNDI. Thermal unfolding was recorded over a temperature range of $20-90^{\circ} \mathrm{C}$ with temperature increase of $5^{\circ} \mathrm{C}$.

C-exNDI displays potent anti-HSV-1 activity. Since our data indicate that even if HSV-1 G4s are not totally preferred, nonetheless they are preferred over telomeric G4s, the most abundant cellular G4 structures in infected cells, we tested the ability of c-exNDI to inhibit HSV-1. In plaque assay, the compound showed 50\% inhibition of HSV-1 production $\left(\mathrm{IC}_{50}\right.$ ) at $18.3 \pm 1.4 \mathrm{nM}$ (Fig. 3a). Interestingly, the compound concentration able to kill $50 \%$ of the cells, as measured by MTT assay, was $628.4 \pm 2.1 \mathrm{nM}$, resulting in a remarkable selectivity index $(\mathrm{SI}=34.3 \pm 2.7)$ (Fig. 3a). To confirm this data, a recombinant HSV-1 expressing GFP fused to the viral protein VP16 (HSV-1 [V41]) was used to infect cells; this mutant virus is characterized by normal replication kinetics and yields ${ }^{32}$. Analysis was performed by flow cytometry. Cells were treated with c-exNDI and acyclovir (ACV), the antiviral drug of choice for treatment of HSV-1 infections ${ }^{33}$, here used as the reference drug to which compare the anti-HSV-1 potency of c-exNDI. Both compounds were used at low/non cytotoxic concentration (i.e. $100 \mathrm{nM}$ c-exNDI and $3 \mu \mathrm{M}$ ACV) corresponding to 5 times their $\mathrm{IC}_{50}$ values ${ }^{34,35}$. Cells infected with HSV-1 [V41] and treated with c-exNDI or ACV at 24 h.p.i were monitored for their GFP fluorescence. C-exNDI (Fig. 3b,c) induced an almost complete reduction of GFP fluorescence (8.6\%, expressed as mean of GFP fluorescence), whereas $26 \%$ of cells remained fluorescent upon treatment with ACV (Fig. 3b,c).

To investigate the mechanism of action of c-exNDI, mRNA levels of representative viral genes were measured at 4 and 24h.p.i. upon treatment with c-exNDI. In particular, genes corresponding to immediate-early (IE: ICP22, ICP47), early (E: UL30) and late (L: UL36) proteins were considered. At 4 h.p.i., no significant inhibition of viral mRNAs production was observed, whereas at 24 h.p.i. all mRNAs were reduced to a similar extent (28-45\%) (Fig. 4a). A similar trend has been reported for $\mathrm{ACV}^{36}$. To assess the main and temporally last viral step targeted 
a

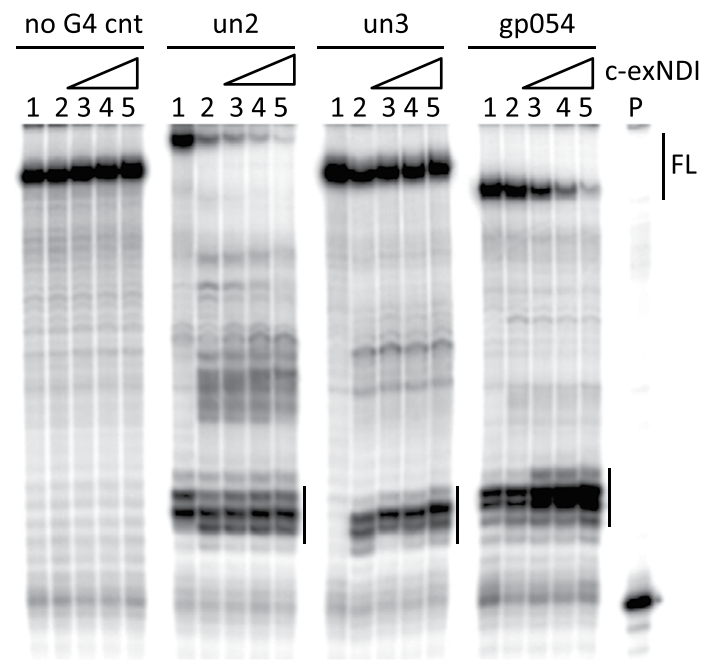

b

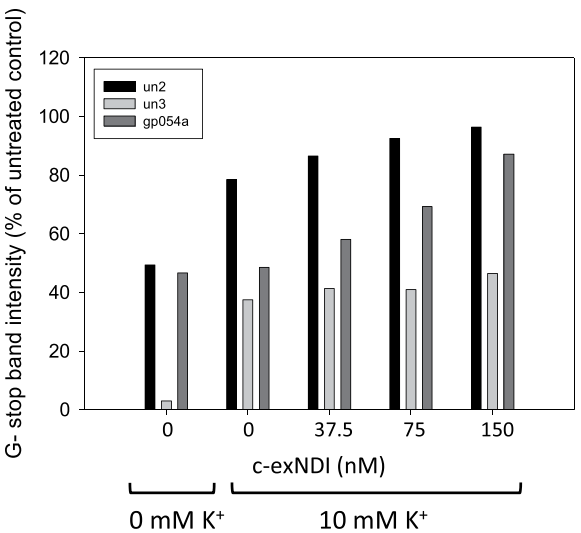

Figure 2. Taq polymerase stop assay on HSV-1 G4 folding sequences in the presence of c-exNDI. (a) un2, un3 and $g p 054 a$ were analyzed in the absence (lanes 1 ) or presence of $10 \mathrm{mM} \mathrm{K}^{+}$(lanes 2-5). c-exNDI was used at concentrations of $37.5,75$ and $100 \mathrm{nM}$ (lanes 3-5). Elongation was performed at $60^{\circ} \mathrm{C}$. A non-G4 folding sequence was used as negative control (no G4 cnt). G4-related stops are indicated by vertical bars. FL stands for "full-length" amplified template. P indicates the primer lane. (b) Quantification of c-exNDI-induced stop sites observed in (a).

\begin{tabular}{|c|c|c|c|c|c|}
\hline \multicolumn{6}{|c|}{ Binding affinity c-exNDI ${ }^{19}$} \\
\hline Competing G4s & un2 & un3 & gp054a & LTR-III & Cell (hTel/myc/kit) \\
\hline un2/LTR-III & 20 & & & 58 & \\
\hline un2/kit & 24 & & & & 46 \\
\hline un $2 / \mathrm{myc}$ & 15 & & & & 68 \\
\hline un2/hTel & 34 & & & & 30 \\
\hline un3/LTR-III & & 43 & & 61 & \\
\hline un3/kit & & 52 & & & 31 \\
\hline un $3 / \mathrm{myc}$ & & 36 & & & 66 \\
\hline un3/hTel & & 72 & & & 21 \\
\hline gp054a/LTR-III & & & 3 & 75 & \\
\hline$g p 054 a /$ kit & & & 7 & & 64 \\
\hline$g p 054 a / \mathrm{myc}$ & & & 4 & & 78 \\
\hline gp054a/hTel & & & 12 & & 39 \\
\hline
\end{tabular}

Table 2. Relative binding affinity, analyzed by MS competition assay for un2, un3, gp054a, hTel21, c-kit2 (kit), c-myc (myc) and HIV-1 LTR-III G4-folded oligonucleotides.

by c-exNDI, we performed a time of addition (TOA) assay where the maximal low/mild-cytotoxic concentration of the compound was added at different times post-infection (corresponding to different viral cycle steps). The compound maintained its inhibitory activity when added up to 8 h.p.i (Fig. $4 \mathrm{~b}$ ). This is the time when viral replication occurs; indeed, ACV, a known inhibitor of the viral DNA polymerase ${ }^{37}$, showed a remarkably overlapping TOA profile. These data indicate that c-exNDI inhibits viral production mainly by targeting viral replication. Inhibition of viral DNA replication was further confirmed by qPCR analysis. At 4 h.p.i no reduction in viral DNA replication and production was observed, whereas at 24 h.p.i viral DNA was reduced of about $40 \%$ (Fig. S1), similarly to what observed with another G4-ligand targeting HSV-1 DNA ${ }^{27}$.

\section{Discussion}

We have shown that c-exNDI, a compound initially developed to specifically interact with HIV-1 G4s, potently stabilizes also G4s found in the HSV-1 genome. Interestingly, even if a net preferentiality for HSV-1 G4s over some cellular G4s was not observed, nevertheless c-exNDI displayed potent anti-HSV-1 activity paralleled by mild/low toxicity at the doses necessary to achieve complete suppression of virus production (Fig. 3a). We ascribe this behaviour to two main reasons: i) the massive presence of G4s during the HSV-1 viral cycle. By an antibody-based approach in cells we demonstrated that viral G4s form massively in the nucleus during viral 

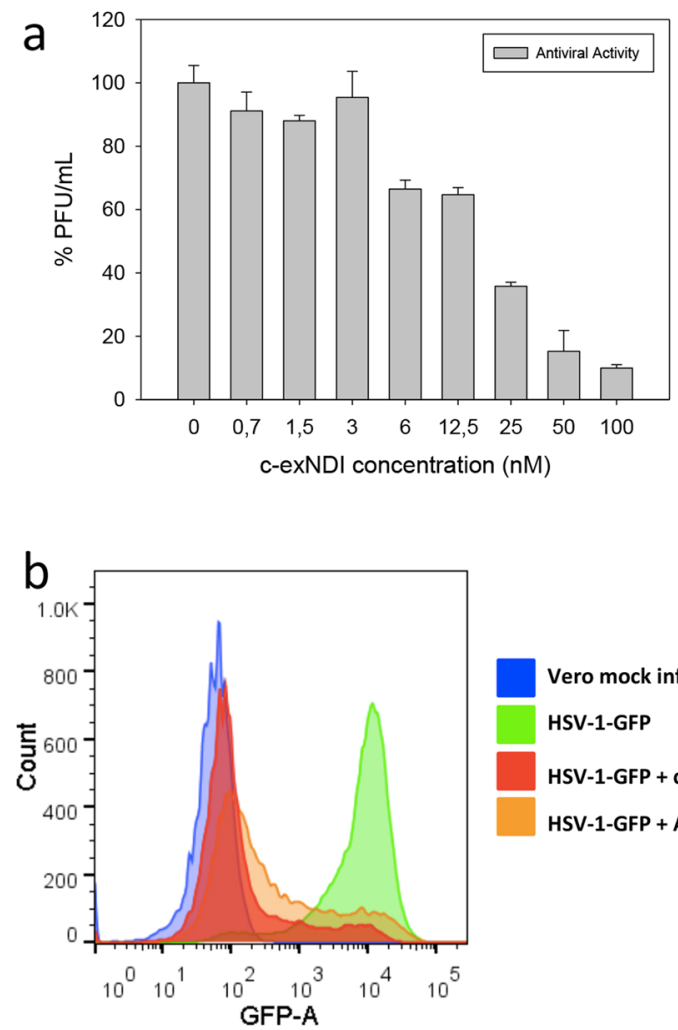

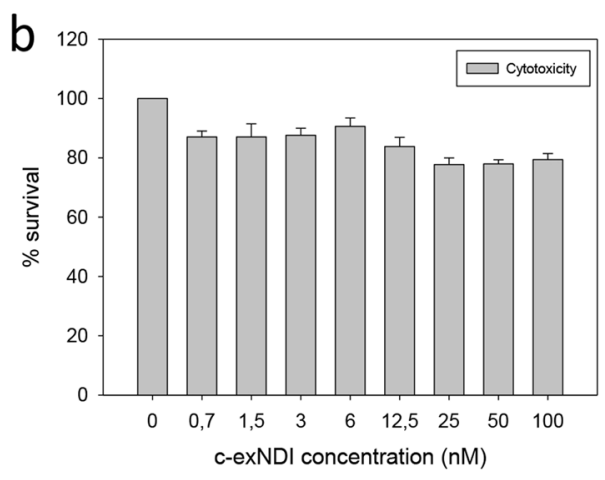

C

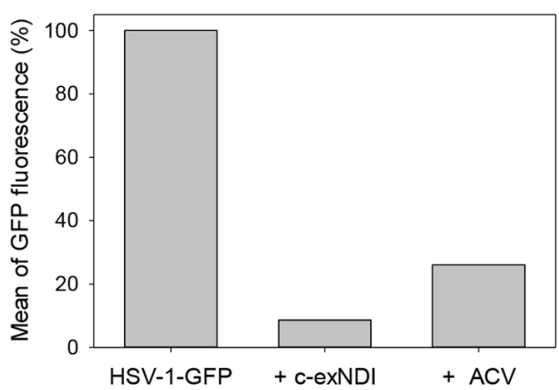

Figure 3. Anti-HSV-1 activity of c-exNDI. (a) Plaque assay: Vero cells were infected with HSV-1 strain F (MOI 1, as previously reported ${ }^{27}$ ) and treated with increasing concentrations of c-exNDI (0.7 nM-100 nM). Supernatants were collected 24 h.p.i. and the number of plaque forming units was determined. (b) Vero cells were treated at the same concentrations of c-exNDI $(0.7 \mathrm{nM}-100 \mathrm{nM})$ used in the antiviral assay and cytotoxicity was evaluated by MTT assay. (c) Flow cytometry of HSV-1 [V41]-infected cells treated with c-exNDI and ACV. HSV-1 [V41]-infected cells were used as positive control and their GFP fluorescence set to $100 \%$. C-exNDI (red curve) and ACV (orange curve)-treated infected cells were compared to HSV-1 [V41]infected cells (green curve) to monitor compound inhibition of GFP fluorescence. (d) Quantification of the mean of GFP fluorescence upon treatment of HSV-1 [V41]-infected cells with c-exNDI and ACV, observed in (b).
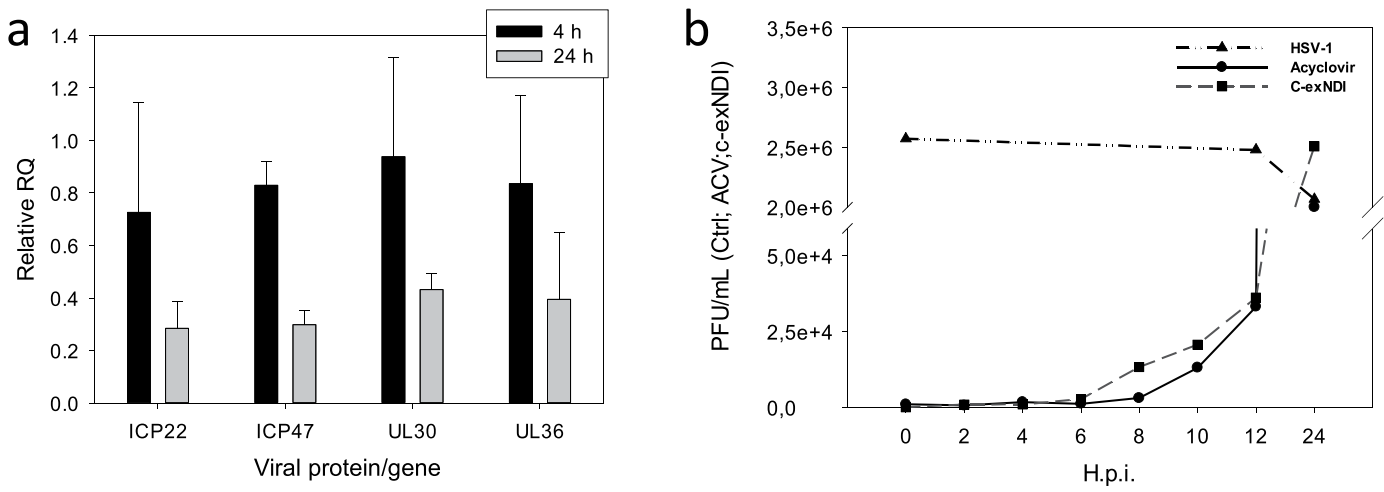

Figure 4. Antiviral effects of c-exNDI. (a) Effect of c-exNDI on mRNA levels of immediate-early (IE), early (E) and late (L) proteins of HSV-1. Infected cells were treated with c-exNDI (100 nM); 4 or 24 h.p.i total RNA was isolated, retrotranscribed into cDNA and expression of specific genes was determined by RT-PCR. RQ are Relative Quantities. Each gene was analyzed in duplicate. In all data sets: $\mathrm{n} \geq 2$, mean \pm s.d., Student's t-test, $\mathrm{p} \leq 0.01$. (b) Effect of c-exNDI on HSV-1 cycle steps evaluated by time of addition assay. C-exNDI was tested at $100 \mathrm{nM}$ (dashed line/squares), acyclovir (ACV) was used as a reference drug and tested at $3 \mu \mathrm{M}$ (solid line/ circles). Data from infected cells treated in the same conditions but without the compounds are reported as dashed line/triangles. Compounds were administered from 0 to 12 h.p.i. and supernatants were collected 30 h.p.i. The left and right Y-axis refer to ACV and c-exNDI data, respectively. 


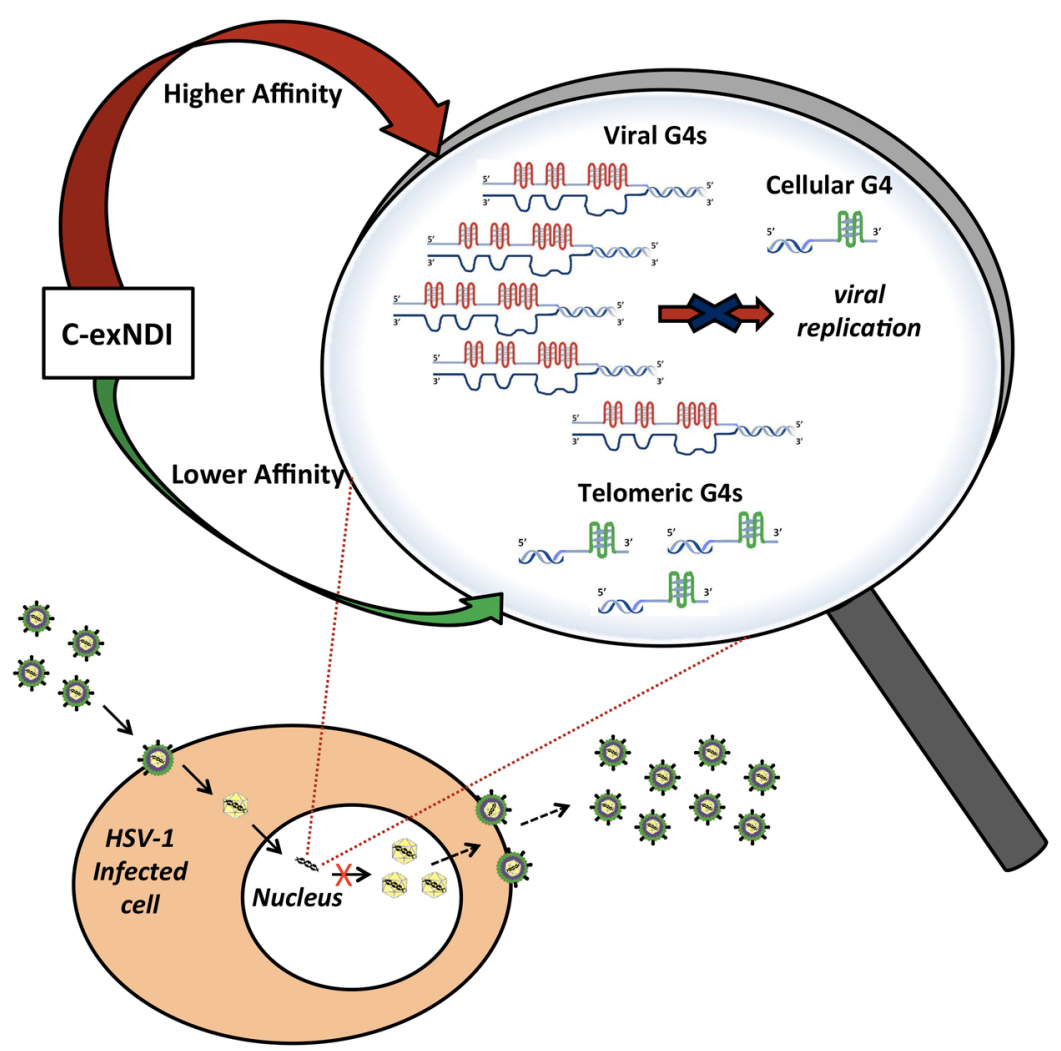

Figure 5. Scheme of the proposed c-exNDI mechanism of anti-HSV-1 activity.

replication ${ }^{28}$. During this step they likely control both viral replication itself and other key processes of the infection, with consequent dramatic effect when they are stabilized by c-exNDI. In fact, viral replication was here shown to be the step targeted by c-exNDI, which strongly points to the compound G4-based mechanism of action on the HSV-1 G4s. ii) The higher affinity of c-exNDI for HSV-1 G4s vs telomeric G4s. These are the most abundant cellular G4s in the infected cell: their lack of optimal recognition by the compound is likely the main reason for the observed low cellular toxicity at antiviral effective doses (Fig. 5).

Impairment of viral replication led to the decrease of viral genes in all phases of the viral life cycle. This effect is shared between c-exNDI and $\mathrm{ACV}^{36}$. We have previously shown that the general G4 ligand, BRACO-19, mainly decreased $\mathrm{L}$ viral genes ${ }^{27}$. This discrepancy may be due to the lower affinity of BRACO-19 for HSV-1 G4s and lower antiviral activity $\left(\mathrm{IC}_{50} \text { in the low micromolar range }\right)^{27}$ which would result in observable inhibition only of genes temporally more closely influenced by inhibition of viral replication. In contrast, in the case of c-exNDI and ACV, their potent effect on the HSV-1 genome is likely sensed at a more extended level. There is also the fascinating possibility that c-exNDI reacts with additional G4s, such as those in key regulatory IE and E genes; in fact, besides the extended G4 repeats in the terminal and inverted repeats ${ }^{27}$, less extended but stable G4s structures are also distributed throughout the HSV-1 genome and embedded in the promoter and coding sequences of fundamental genes ${ }^{27}$.

The unique mechanism of action of c-exNDI makes it suitable for treatment of HSV-1 strains resistant to current therapies; for instance, the emergence of ACV resistant strains has long created an obstacle for the treatment of HSV- $1^{38}$. Even though c-exNDI has a less wide therapeutic window than ACV, it is active at nanomolar concentrations, a promising feature for its prospect development as anti-herpetic drugs. Since the HSV-1 infection is a common illness associated to immunosuppression, the impact of our data extends to AIDS, cancer and transplanted patients.

\section{Methods}

Cells and viruses. Vero cells (Sigma-Aldrich, Saint Louis, USA) were grown in DMEM supplemented with 10\% FBS and 1X PenStrep antibiotic (Gibco, Life Technologies, Monza, Italy). HSV-1 strain F was a kind gift of B. Roizman (University of Chicago, Illinois, USA). Recombinant HSV-1 expressing VP16-GFP (HSV-1 [V41]) was kindly provided by Peter O’ Hare (Imperial College, London, UK).

Oligonucleotides and compounds. All oligonucleotides and primers were from Sigma Aldrich (Milan, Italy), Table S1. The G4 ligand c-exNDI was synthesized and provided by Prof. M. Freccero (University of Pavia, Italy). The control compound acyclovir (ACV) was purchased from Sigma Aldrich (Milan, Italy). 
Circular Dichroism. Circular dichroism (CD) experiments were performed using a Chirascan-Plus (Applied Photophysics, Leatherhead, UK) equipped with a Peltier temperature controller using a quartz cell of $5 \mathrm{~mm}$ path length. G4 folding oligonucleotides were diluted to a final concentration of $4 \mu \mathrm{M}$ in the absence or presence $(100 \mathrm{mM})$ of $\mathrm{KCl}$ and $10 \mathrm{mM}$ lithium cacodilate buffer. After annealing step $\left(5 \mathrm{~min}\right.$ at $\left.95^{\circ} \mathrm{C}\right)$, DNA samples were gradually cooled down and, where specified, c-exNDI was added at a final concentration of $16 \mu \mathrm{M}$. Thermal unfolding analyses were recorded from 230 to $320 \mathrm{~nm}$ over a temperature range of $20-90^{\circ} \mathrm{C}\left(5^{\circ} \mathrm{C} / \mathrm{min}\right)$. The reported spectrum of each sample represents the average of 2 scans and is baseline-corrected for buffer contribution. Observed ellipticities were converted to mean residue ellipticity $(\theta)=\operatorname{deg} \times \mathrm{cm}^{2} \times \mathrm{dmol}^{-1}$ (mol ellip). $\mathrm{T}_{\mathrm{m}}$ values were calculated according to the Van't Hoff equation, applied for a two-state transition, assuming that the heat capacity of the folded and unfolded states are equal.

Taq polymerase stop assay. The Taq polymerase stop assay was performed as previously described ${ }^{5}$. Briefly, the $5^{\prime}$-end labeled primer (HSV Taq primer, Table S1) was annealed to each template (Table S1) in lithium cacodylate buffer. Where specified, samples were incubated with $\mathrm{KCl} 10 \mathrm{mM}$ in the presence or absence of c-exNDI $(0,37.5,75,150 \mathrm{nM})$ at rt. Elongation was carried out at $60^{\circ} \mathrm{C}$ for 30 min using $2 \mathrm{U}$ of AmpliTaq Gold DNA polymerase (Applied Biosystem, Carlsbad, California, USA). All reactions were stopped by ethanol precipitation and primers elongation products were resolved on a $15 \%$ polyacrylamide denaturing gel. Gel visualization was performed with phosphoimaging (Typhoon FLA 9000, GE Healthcare, Milan, Italy).

Mass Spectrometric (MS) competition assay. Oligonucleotides were heat-denatured and folded in $0.8 \mathrm{mM} \mathrm{KCl}, 120 \mathrm{mM}$ trimethylammonium acetate (TMAA), $\mathrm{pH} 7.0$, and 20\% isopropanol (IPA) overnight at $4{ }^{\circ} \mathrm{C}$. Oligonucleotides were diluted to the final concentration of $4 \mu \mathrm{M}$ and incubated with c-exNDI at DNA:compound $1: 1$ overnight at $4^{\circ} \mathrm{C}$. Samples were analyzed by direct infusion electrospray ionization (ESI) on a Xevo G2-XS QTof mass spectrometer (Waters, Manchester, UK). The injection was automatically performed by an Agilent 1290 Infinity HPLC (Agilent Technologies, Santa Clara, CA, US) equipped with an auto sampler; the carrying buffer was TMAA $80 \mathrm{mM}, 20 \%$ IPA. Up to $5 \mu \mathrm{L}$ samples were typically injected per each analysis. The ESI source settings were the following: electrospray capillary voltage set at $1.8 \mathrm{kV}$, the source and desolvation temperatures were $45^{\circ} \mathrm{C}$ and $65^{\circ} \mathrm{C}$ respectively, the sampling cone was set at $65 \mathrm{~V}$. All these parameters ensured minimal fragmentation of the DNAs complexes. The instrument was calibrated using a $2 \mathrm{mg} / \mathrm{mL}$ solution of sodium iodide in $50 \%$ of IPA. Binding affinities were calculated for each experiment using the reconstructed-ion chromatogram area for each species calculated by MassLynx V4.1. The binding affinity was calculated with the following formula: $[\mathrm{BA}=(\Sigma \mathrm{G} 4 \mathrm{~b} /(\Sigma \mathrm{G} 4 \mathrm{f}+\Sigma \mathrm{G} 4 \mathrm{~b})) \times 100]$, where BA is the binding affinity, G4b is chromatogram area of bound G4 DNA, and G4f is the chromatogram area of free G4 DNA.

Cytotoxicity assay. Cytotoxicity of c-exNDI in Vero cells was determined by MTT assay. Briefly, serial dilutions of c-exNDI $(0.7-12.8 \mu \mathrm{M})$ were dispensed to $24 \mathrm{~h}$ plated Vero cells $\left(1 \times 10^{4}\right.$ cells/well $)$. After $48 \mathrm{~h}$ from treatment, cells were supplemented with freshly diluted 3-(4,5-dimethylthiazol-2-yl)-2,5-diphenyltetrazolium bromide (MTT) (Sigma-Aldrich, Milan, Itlay) solution $(5 \mathrm{mg} / \mathrm{mL})$ and incubated for $4 \mathrm{~h}$. After solubilization, absorbance was measured by Sunrise Tecan plate reader (Mannendorf, Switzerland) at $620 \mathrm{~nm}$. The $50 \%$ cytotoxic concentration $\left(\mathrm{CC}_{50}\right)$ was determined from the dose-response curve.

Antiviral assays. The antiviral activity of c-exNDI against HSV-1 (F) was investigated by plaque assay. For virus infection, wild- type (wt) HSV-1 (F) was added to Vero cells at different multiplicities of infection (MOIs) in serum-free medium. After $1 \mathrm{~h}$ at $37^{\circ} \mathrm{C}$, the inoculums were replaced with complete medium. After infection, the compound was added at increasing concentrations (0.7-100 nM) in each well. Because a single round of HSV-1 replication takes around $24 \mathrm{~h}$ to complete and at this time p.i. the production of virus reaches a plateau ${ }^{39,40}$, supernatants were collected 24 h.p.i and stored at $-80^{\circ} \mathrm{C}$ until viral titrations by plaque assay. For plaque reduction assay, Vero cells were seeded in 24 -well plate $\left(1 \times 10^{5} /\right.$ well $)$ and incubated overnight. Cells were then infected with $250 \mu \mathrm{l}$ of serially-diluted (10-folds) supernatants for $1 \mathrm{~h}$ at $37^{\circ} \mathrm{C}$. After infection, cells were washed with PBS $1 \mathrm{X}$ and incubated with $500 \mu \mathrm{l}$ of DMEM supplemented with $0.6 \%$ methylcellulose and $2 \%$ FBS. Forty-eight h.p.i., cells were washed with PBS 1X and fixed with formaldehyde 5\% in PBS 1X for $10 \mathrm{~min}$ at room temperature, then colored with crystal violet $0.8 \%$ (in ethanol $50 \%$ ). Viral plaques were counted using an optical microscope (Zeiss, Jena, Germany).

Time of addition assay. Time of addition assay (TOA) was performed to establish which is the last step of the viral infection cycle affected by the compound ${ }^{41}$. Vero cells were seeded in 24 -well plate $\left(1 \times 10^{5}\right.$ cells/well $)$ and incubated overnight. Cells were then infected with HSV-1 (F) at a MOI of 0.5 (as suggested ${ }^{41}$ ) and treated every two hours (from 0 to $12 \mathrm{~h} . \mathrm{p} . \mathrm{i})$ with c-exNDI $(100 \mathrm{nM})$ or ACV $(3 \mu \mathrm{M})$ as reference drug. To allow estimation of the c-exNDI effects at all viral steps, supernatants were collected 30 h.p.i. and then titrated following plaque assay working protocol as described above.

Flow cytometry. Vero cells were seeded into 12 -wells plates $\left(1 \times 10^{5}\right.$ cells/well $)$ and next day infected with mutant HSV-1 [V41] expressing VP16-GFP using the procedure described above. MOI of 0.3 was used because at higher MOIs the GFP signal reached saturation levels. After infection, Vero cells were incubated for $24 \mathrm{~h}$ with complete medium or with complete medium containing c-exNDI $(100 \mathrm{nM})$ or ACV $(3 \mu \mathrm{M})$. GFP fluorescence was acquired for 30.000 events in each sample using FACS Cytofluorometer BD LSR II (BD Bioscences, New Jersey, USA). Fluorescence acquisitions were analyzed with FlowJo software (Tree Star, Oregon, USA).

Real-time PCR and qPCR. For total RNA extraction Vero cells were plated $\left(1.8 \times 10^{5} /\right.$ well $)$, mock- or HSV-1 (F)-infected (MOI of 3) and treated with c-exNDI 100 nM. At 4 or 24h.p.i. total RNA was isolated using 
TRIzol reagent (Life Technologies, Monza, Italy) according to the manufacturer's instructions and subjected to RNase free DNase I treatment (Ambion Turbo DNA free, Life Technologies, Monza, Italy). Extracted RNA $(1 \mu \mathrm{g})$ was subjected to reverse transcription by $1.5 \mathrm{U}$ MuLv (Life Technologies, Monza, Italy). Reverse transcription was carried out as follows: $10 \mathrm{~min}$ at $25^{\circ} \mathrm{C}, 60 \mathrm{~min}$ at $48^{\circ} \mathrm{C}$ and $5 \mathrm{~min}$ at $95^{\circ} \mathrm{C}$ using the Thermo Cycler Verity 96 (Applied Biosystem, Monza, Italy). Forward/reverse primers were designed within conserved HSV-1 gene sequences using Primer Express 3 (Applied Biosystem, Monza, Italy) Table S2. Real-time PCR was performed using TaqMan chemistry with 5'-[FAM] and 3'-[TAMRA] end labeled probes. Realtime reaction was composed of FAST Master Mix 2X (Applied Biosystem, Monza, Italy), $900 \mathrm{nM}$ of forward/reverse primer mixture, $200 \mathrm{nM}$ of TaqMan probe, sterile water and $3 \mu \mathrm{L}$ of cDNA. Experiments were performed using ABI $7900 \mathrm{HT}-\mathrm{FAST}$ RealTime PCR System under the following conditions: $95^{\circ} \mathrm{C}$ for $10 \mathrm{~min}$ followed by 40 cycles of 30 seconds at $95^{\circ} \mathrm{C}$ and 30 seconds at $58^{\circ} \mathrm{C}, 1$ minute at $72^{\circ} \mathrm{C}$. Messenger RNA transcription levels were standardized against the housekeeping gene $\beta$-actin. Non- treated retrotranscribed HSV-1 RNA was used as mRNA expressed control. Each sample was analyzed in duplicate.

For quantitative PCR (qPCR) cells were plated (1.8 $\times 10^{5} /$ well), mock- or HSV-1 (F)-infected (MOI of 1) and treated with c-exNDI at $100 \mathrm{nM}$. At 4 or 24 h.p.i. cells were collected and total DNA was extracted using NucleoSpin Tissue (Macherey- Nagel, Duren, Germany) according to the manufacturer's instruction. Isolated intracellular DNA was analyzed by realtime PCR using TaqMan chemistry with $5^{\prime}$-[FAM] and $3^{\prime}$-[TAMRA]-end labeled probes (Table S2) in a final volume of $25 \mu \mathrm{L}$. qPCR reaction was composed of FAST Master Mix 2X (Applied Biosystem, Monza, Italy), $500 \mathrm{nM}$ of forward/reverse primer mixture (gene US1, Table S2), $200 \mathrm{nM}$ of TaqMan probe, sterile water and $10 \mu \mathrm{L}$ of isolated DNA. Experiments were performed using ABI $7900 \mathrm{HT}-\mathrm{FAST}$ RealTime PCR System under the following conditions: $95^{\circ} \mathrm{C}$ for $5 \mathrm{~min}$ followed by 45 cycles of 5 seconds at $95^{\circ} \mathrm{C}$ and 10 seconds at $60^{\circ} \mathrm{C}$. Each sample was analyzed in duplicate.

\section{References}

1. Lipps, H. J. \& Rhodes, D. G-quadruplex structures: in vivo evidence and function. Trends in cell biology 19, 414-422, doi:10.1016/j. tcb.2009.05.002 (2009).

2. Sen, D. \& Gilbert, W. A sodium-potassium switch in the formation of four-stranded G4-DNA. Nature 344, 410-414, doi:10.1038/344410a0 (1990).

3. Rhodes, D. \& Lipps, H. J. G-quadruplexes and their regulatory roles in biology. Nucleic acids research 43, 8627-8637, doi:10.1093/ nar/gkv862 (2015).

4. Zhou, B., Liu, C., Geng, Y. \& Zhu, G. Topology of a G-quadruplex DNA formed by C9orf72 hexanucleotide repeats associated with ALS and FTD. Scientific reports 5, 16673, doi:10.1038/srep16673 (2015).

5. Perrone, R. et al. A dynamic G-quadruplex region regulates the HIV-1 long terminal repeat promoter. J Med Chem 56, 6521-6530, doi:10.1021/jm400914r (2013).

6. Holder, I. T. \& Hartig, J. S. A matter of location: influence of G-quadruplexes on Escherichia coli gene expression. Chem Biol 21, 1511-1521, doi:10.1016/j.chembiol.2014.09.014 (2014).

7. Maizels, N. G4-associated human diseases. EMBO Rep 16, 910-922, doi:10.15252/embr.201540607 (2015).

8. Fry, M. \& Loeb, L. A. The fragile X syndrome d(CGG)n nucleotide repeats form a stable tetrahelical structure. Proc Natl Acad Sci USA 91, 4950-4954 (1994).

9. Sket, P. et al. Characterization of DNA G-quadruplex species forming from C9ORF72 G4C2-expanded repeats associated with amyotrophic lateral sclerosis and frontotemporal lobar degeneration. Neurobiol Aging 36, 1091-1096, doi:10.1016/j. neurobiolaging.2014.09.012 (2015).

10. Qiu, J. et al. Biological Function and Medicinal Research Significance of G-Quadruplex Interactive Proteins. Curr Top Med Chem 15, 1971-1987 (2015).

11. Tosoni, E. et al. Nucleolin stabilizes G-quadruplex structures folded by the LTR promoter and silences HIV-1 viral transcription. Nucleic acids research 43, 8884-8897, doi:10.1093/nar/gkv897 (2015).

12. Henderson, A. et al. Detection of G-quadruplex DNA in mammalian cells. Nucleic acids research 42, 860-869, doi:10.1093/nar/ gkt957 (2014).

13. Biffi, G., Tannahill, D., McCafferty, J. \& Balasubramanian, S. Quantitative visualization of DNA G-quadruplex structures in human cells. Nat Chem 5, 182-186, doi:10.1038/nchem.1548 (2013).

14. Metifiot, M., Amrane, S., Litvak, S. \& Andreola, M. L. G-quadruplexes in viruses: function and potential therapeutic applications. Nucleic acids research 42, 12352-12366, doi:10.1093/nar/gku999 (2014).

15. Amrane, S. et al. Topology of a DNA G-quadruplex structure formed in the HIV-1 promoter: a potential target for anti-HIV drug development. Journal of the American Chemical Society 136, 5249-5252, doi:10.1021/ja501500c (2014).

16. Perrone, R. et al. Formation of a unique cluster of G-quadruplex structures in the HIV-1 Nef coding region: implications for antiviral activity. PLoS One 8, e73121, doi:10.1371/journal.pone.0073121 (2013).

17. Piekna-Przybylska, D., Sullivan, M. A., Sharma, G. \& Bambara, R. A. U3 region in the HIV-1 genome adopts a G-quadruplex structure in its RNA and DNA sequence. Biochemistry 53, 2581-2593, doi:10.1021/bi4016692 (2014).

18. Perrone, R. et al. Anti-HIV-1 activity of the G-quadruplex ligand BRACO-19. J Antimicrob Chemother 69, 3248-3258, doi:10.1093/ $\mathrm{jac} / \mathrm{dku} 280$ (2014).

19. Perrone, R. et al. Synthesis, Binding and Antiviral Properties of Potent Core-Extended Naphthalene Diimides Targeting the HIV-1 Long Terminal Repeat Promoter G-Quadruplexes. J Med Chem 58, 9639-9652, doi:10.1021/acs.jmedchem.5b01283 (2015).

20. Tan, J. et al. The SARS-unique domain (SUD) of SARS coronavirus contains two macrodomains that bind G-quadruplexes. PLoS Pathog 5, e1000428, doi:10.1371/journal.ppat.1000428 (2009).

21. Tluckova, K. et al. Human papillomavirus G-quadruplexes. Biochemistry 52, 7207-7216, doi:10.1021/bi400897g (2013).

22. Wang, S. R. et al. A highly conserved G-rich consensus sequence in hepatitis C virus core gene represents a new anti-hepatitis C target. Sci Adv 2, e1501535, doi:10.1126/sciadv.1501535 (2016).

23. Fleming, A. M., Ding, Y., Alenko, A. \& Burrows, C. J. Zika Virus Genomic RNA Possesses Conserved G-Quadruplexes Characteristic of the Flaviviridae Family. ACS infectious diseases 2, 674-681, doi:10.1021/acsinfecdis.6b00109 (2016).

24. Wang, S. R. et al. Chemical Targeting of a G-Quadruplex RNA in the Ebola Virus L Gene. Cell chemical biology 23, 1113-1122, doi:10.1016/j.chembiol.2016.07.019(2016).

25. Murat, P. et al. G-quadruplexes regulate Epstein-Barr virus-encoded nuclear antigen 1 mRNA translation. Nat Chem Biol 10, 358-364, doi:10.1038/nchembio.1479 (2014).

26. Norseen, J., Johnson, F. B. \& Lieberman, P. M. Role for G-quadruplex RNA binding by Epstein-Barr virus nuclear antigen 1 in DNA replication and metaphase chromosome attachment. Journal of virology 83, 10336-10346, doi:10.1128/jvi.00747-09 (2009).

27. Artusi, S. et al. The Herpes Simplex Virus-1 genome contains multiple clusters of repeated G-quadruplex: Implications for the antiviral activity of a G-quadruplex ligand. Antiviral research 118, 123-131, doi:10.1016/j.antiviral.2015.03.016 (2015). 
28. Artusi, S. et al. Visualization of DNA G-quadruplexes in herpes simplex virus 1-infected cells. Nucleic acids research 44, 10343-10353, doi:10.1093/nar/gkw968 (2016).

29. Xiong, Y. X., Huang, Z. S. \& Tan, J. H. Targeting G-quadruplex nucleic acids with heterocyclic alkaloids and their derivatives. Eur J Med Chem 97, 538-551, doi:10.1016/j.ejmech.2014.11.021 (2015).

30. Ambrus, A., Chen, D., Dai, J., Jones, R. A. \& Yang, D. Solution structure of the biologically relevant G-quadruplex element in the human c-MYC promoter. Implications for G-quadruplex stabilization. Biochemistry 44, 2048-2058, doi:10.1021/bi048242p (2005).

31. Hsu, S. T. et al. A G-rich sequence within the c-kit oncogene promoter forms a parallel G-quadruplex having asymmetric G-tetrad dynamics. Journal of the American Chemical Society 131, 13399-13409, doi:10.1021/ja904007p (2009).

32. La Boissiere, S., Izeta, A., Malcomber, S. \& O'Hare, P. Compartmentalization of VP16 in cells infected with recombinant herpes simplex virus expressing VP16-green fluorescent protein fusion proteins. Journal of virology 78, 8002-8014, doi:10.1128/ JVI.78.15.8002-8014.2004 (2004).

33. Vere Hodge, R. A. \& Field, H. J. Antiviral agents for herpes simplex virus. Advances in pharmacology 67, 1-38, doi:10.1016/B978-012-405880-4.00001-9 (2013).

34. Pavic, I. et al. Flow cytometric analysis of herpes simplex virus type 1 susceptibility to acyclovir, ganciclovir, and foscarnet. Antimicrobial agents and chemotherapy 41, 2686-2692 (1997).

35. Piret, J., Goyette, N. \& Boivin, G. Novel Method Based on Real-Time Cell Analysis for Drug Susceptibility Testing of Herpes Simplex Virus and Human Cytomegalovirus. Journal of clinical microbiology 54, 2120-2127, doi:10.1128/JCM.03274-15 (2016).

36. Zheng, K. et al. Inhibition of herpes simplex virus type 1 entry by chloride channel inhibitors tamoxifen and NPPB. Biochemical and biophysical research communications 446, 990-996, doi:10.1016/j.bbrc.2014.03.050 (2014).

37. Elion, G. B. Acyclovir: discovery, mechanism of action, and selectivity. J Med Virol 1 Suppl, 2-6 (1993).

38. Bacon, T. H., Levin, M. J., Leary, J. J., Sarisky, R. T. \& Sutton, D. Herpes simplex virus resistance to acyclovir and penciclovir after two decades of antiviral therapy. Clinical microbiology reviews 16, 114-128 (2003).

39. Lehman, I. R. \& Boehmer, P. E. Replication of herpes simplex virus DNA. The Journal of biological chemistry 274, 28059-28062 (1999).

40. Lester, J. T. \& DeLuca, N. A. Herpes simplex virus 1 ICP4 forms complexes with TFIID and mediator in virus-infected cells. Journal of virology 85, 5733-5744, doi:10.1128/JVI.00385-11 (2011).

41. Daelemans, D., Pauwels, R., De Clercq, E. \& Pannecouque, C. A time-of-drug addition approach to target identification of antiviral compounds. Nat Protoc 6, 925-933, doi:10.1038/nprot.2011.330 (2011).

\section{Acknowledgements}

This work was supported by the Bill and Melinda Gates Foundation (GCE grant numbers OPP1035881, OPP1097238) and the European Research Council (ERC Consolidator grant 615879) to SNR. Funding for open access charge: Bill and Melinda Gates Foundation. We thank Prof. M. Freccero for sharing c-exNDI and helpful discussion.

\section{Author Contributions}

S.C. performed the spectroscopic analysis and the antiviral assays and contributed to write the manuscript; R.P. performed the Taq polymerase stop assay and flow cytometry analysis and contributed to write the manuscript; M.S. performed the mass spectrometry competition assay; F.D. synthesized c-exNDI; G.P. discussed the data; S.N.R. conceived of the study, analyzed the data and wrote the manuscript. All authors analyzed the data and reviewed the manuscript.

\section{Additional Information}

Supplementary information accompanies this paper at doi:10.1038/s41598-017-02667-3

Competing Interests: The authors declare that they have no competing interests.

Publisher's note: Springer Nature remains neutral with regard to jurisdictional claims in published maps and institutional affiliations.

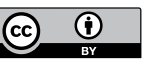

Open Access This article is licensed under a Creative Commons Attribution 4.0 International License, which permits use, sharing, adaptation, distribution and reproduction in any medium or format, as long as you give appropriate credit to the original author(s) and the source, provide a link to the Creative Commons license, and indicate if changes were made. The images or other third party material in this article are included in the article's Creative Commons license, unless indicated otherwise in a credit line to the material. If material is not included in the article's Creative Commons license and your intended use is not permitted by statutory regulation or exceeds the permitted use, you will need to obtain permission directly from the copyright holder. To view a copy of this license, visit http://creativecommons.org/licenses/by/4.0/.

(C) The Author(s) 2017 